\title{
INFANCIA Y ADOLESCENCIA MAPUCHE EN RELATOS DE LA ARAUCANÍA
}

\author{
Guillermo Williamson \\ Isolde Pérez \\ Francisca Modesto \\ Universidad de La Frontera (Chile) \\ Guillermo Coilla \\ Ministerio de Educación (Chile) \\ Nilsa Raín \\ Universidad Autónoma de Madrid
}

\begin{abstract}
RESUMEN: La infancia indígena y en ella la indígena mapuche es casi invisible en la literatura especializada de educación intercultural bilingüe y en general de la educación. Una revisión de textos de autores mapuche y viajeros no mapuche antiguos y de historiadores y educadores actuales, dan pistas y pautas para describir y entender la infancia y adolescencia mapuche actual. Este pueblo originario se localiza en campos y ciudades de Chile y especialmente en el sur del país, en la Región de La Araucanía. El artículo recoge el testimonio de sabios mapuche y viajeros extranjeros que narran sus visiones sobre la niñez mapuche de su época. Se hace, además, una revisión de algunos estudios actuales referidos al tema. El artículo presenta y reflexiona sobre estas visiones como contribución a una mejor comprensión de los niños, niñas y jóvenes mapuche que participan del proceso pedagógico de la educación intercultural bilingüe.
\end{abstract}

PALABRAS CLAVE: Infancia, adolescencia, Mapuche, educación intercultural bilingüe

\section{INFANCY AND ADOLESCENCE MAPUCHE IN LITERATURE OF THE ARAUCANÍA}

ABSTRACT: The indigenous infancy and in her indigenous Mapuche is almost invisible in the specialized literature of intercultural bilingual education 
and in general of the education. A review of texts of authors Mapuche and travelers not Mapuche ancient and of historians and current educators, they give tracks and guidelines to describe and to understand the infancy and adolescence current Mapuche. This original people is located in fields and cities of Chile and specially in the south of the country, in the Region of The Araucanía. The article the wise persons' testimony gathers Mapuche and travelling foreigners who narrate his visions on the childhood Mapuche of his epoch. There is done, in addition, a review of some current studies referred to the topic. The article presents and thinks about these visions as contribution to a better comprehension of the children, girls and young women Mapuche who take part of the pedagogic process of the intercultural bilingual education.

KEYWORDS: Infancy, adolescente, Mapuche, intercultural bilingual education.

Recibido: $26 / 04 / 2011$

Aceptado: 25/11/2011

\section{INTRODUCCIÓN}

Estudios sobre infancia mapuche son escasos, tanto en Chile como Argentina y en general en la comunidad académica. Hay publicaciones de diversa índole que, más que referirse a los niños, niñas y adolescentes, lo hacen respecto de acciones para ellos. Al colocar "infancia mapuche" en algún buscador de internet aparece una importante cantidad de citas que, sin embargo, adolecen de lo mismo: se refieren a "programas" hacia los niños, más que tratar de ellos. Esto muestra que es necesario investigar más sobre el tema desde los diversos ámbitos del conocimiento. La literatura no es abundante en señalar en forma explícita cómo se desarrollaban los patrones de formación y socialización de la infancia y adolescencia en el pueblo mapuche. Hoy gracias a algunos escasos trabajos, es posible encontrar testimonios de personas mapuche que hablan de su infancia; la acumulación de conocimiento sistemático, producto de investigaciones, también es reducida. Ello es señal de la invisibilidad de la infancia indígena y de su reciente aparición histórica en las preocupaciones académicas, científicas e intelectuales. Se revisan textos de testigos y viajeros mapuche y no mapuche antiguos y de historiadores y educadores actuales, que dan pistas y pautas para entender la infancia y adolescencia mapuche. En Chile las políticas de educación intercultural bilingüe (EIB) han definido en los últimos años varias acciones referidas a estimular la integración cultural y lingüística mapuche en las escuelas rurales básicas (Decreto Supremo del Ministerio de Educación $N^{\circ} 280,2009$ ). Ello se realiza en un marco referencial en que las características de la infancia y la adolescencia mapuche no forman parte de las consideraciones políticas y programáticas: el énfasis está en la formación y acreditación de educadores tradicionales (Kimeltuchefe), los programas de estudio mapuche, los materiales de enseñanza, las relaciones entre educadores profesionales y tradicionales. La infancia y adolescencia continúa invisible. El artículo reflexiona desde diversos testimonios y estudios como contribución a una mejor comprensión de los niños, niñas y 
jóvenes mapuche que participan del proceso educativo formal y que constituyen el sentido principal de la EIB y en general aporta a la comprensión curricular y pedagógica de escenarios educativos desde un enfoque societal multicultural.

\title{
1. LA INFANCIA MAPUCHE EN LA LITERATURA
}

Este capítulo realiza un recorrido lineal de tres textos de distintas épocas que en alguno de sus capítulos hacen referencias respecto de la infancia y adolescencia mapuche, asociadas a procesos familiares y comunitarios de formación que conformaban su vida. Todos los testimonios se realizan en una noción territorial amplia de La Araucanía que abarcaba una extensa zona sureña que representó un no-Chile hasta la conformación hoy como una región institucionalizada. Uno de un conquistador español del 1.500, un segundo de un científico viajero polaco llegado a Chile y un Ionco $^{1}$ mapuche de La Araucanía en el paso del Siglo XIX al XX. El primero observa en un estado inicial la educación, los últimos cuando el territorio ya se había integrado forzosamente al estado nacional chileno y transitaba hacia una organización y articulación territorial del país y la escuela se extendía como el sistema educacional público y nacional por La Araucanía.

\subsection{Alonso de Ercilla y Zúñiga}

En la literatura de los conquistadores españoles es posible encontrar algunas señales de la vida de los niños. En primer lugar, destaca La Araucana, poema de Alonso de Ercilla y Zúñiga (1533-1594)², donde, por su carácter épico, al narrar la Guerra de Arauco se centra en la formación militar de los niños; es decir, en el proceso de preparación del adulto que debe enfrentar al conquistador. Dice en el Canto I (Ercilla y Zúñiga, 1997):

\author{
En lo que usan los niños en teniendo \\ habilidad y fuerza provechosa, \\ es que un trecho seguido ha de ir corriendo \\ por un áspera cuesta pedregosa \\ y al puesto y fin del curso revolviendo, \\ le dan al vencedor alguna cosa. \\ Vienen a ser tan sueltos y alentados \\ que alcanzan por aliento los venados. \\ Y desde la niñez al ejercicio \\ los apremian por fuerza y los incitan, \\ y en el bélico estudio y duro oficio, \\ entrando en más edad, los ejercitan.
}

1. En mapuzungun "cabeza". Jefe de una comunidad mapuche con responsabilidades administrativas, políticas y religiosas.

2. Ercilla regresa a España y la publica en Madrid en tres partes durante dos décadas. El 1 er volumen se editó en 1569 (y una $2^{a}$. edición en 1574), el $2^{\circ}$ en 1578 y el $3^{\circ}$ en 1589. 
Si alguno de flaqueza da un indicio, del uso militar lo inhabilitan, y el que sale en las armas señalado conforme a su valor le dan el grado. (...)

Cada soldado una arma solamente ha de aprender, y en ella ejercitarse, y es aquella a que más naturalmente en la niñez mostrare aficionarse.

Ercilla muestra -en un contexto de guerra y en su visión épica- la preocupación mapuche por formar desde la infancia los guerreros que se requerían para enfrentar a los invasores de su territorio. Estos versos muestran las características fundamentales de los procesos educativos mapuche o de algunos de ellos en ciertos contextos históricos. Implican el desarrollo físico a través del ejercicio, con incentivos al mejor, centrado en la especialización del uso de un arma al que, desde su niñez y en forma natural se aficiona más. Aquel que por diversos motivos mostrase debilidad, era excluido del proceso formativo y probablemente entrenado en otras habilidades no directamente guerreras. Se trata de descubrir y desarrollar las habilidades de cada niño, desde la temprana infancia y de acuerdo a sus intereses, que emergen naturalmente, según los intereses del pueblo mapuche.

La educación es sistemática pero no institucional, se ordena según el interés colectivo y la condición político-histórica coyuntural, forma en una especialidad adecuada al interés y gusto natural del niño coherente con su Az (carácter de la personalidad por una vocación natural), con una pedagogía de prácticas progresivas auténticas, con una preocupación por el desarrollo físico, con refuerzos e incentivos para los que adquieren mejores competencias y relocalización formativa para quienes no alcanzan los logros previstos y necesarios.

\subsection{Lonco Pascual Coña}

A fines del siglo XIX el lonco Pascual Coña, en Testimonio de un cacique mapuche, presenta algunos indicios que permiten conocer parte de la vida y formación de la infancia mapuche en la transición del siglo XIX al XX. (Pascual Coña, Lonco, $1995)^{3}$. La crianza se establece en directa relación con la madre, quien muestra un gran cuidado por los niños. Pascual Coña describe detalladamente la cuna de su raza y cómo se va envolviendo y protegiendo al bebé y, a través de ello, hace referencia al modo de crianza. "La cuna con la guagua queda arrimada a la pared de la casa. El niño que está parado en su cuna puede ver a su madre y a todas las personas que trajinan por la casa, con eso se sosiega. Si tiene sueño se le pone en su cuna sobre el catre y luego duerme profundamente. En el caso que llore, se le mece o se le tira por encima del suelo en su cuna, arrastrando la cuna sobre sus patas poste-

3. El testimonio, dictado en 1925 y 1926 al padre capuchino Ernesto Wilheim de Moesbach, fue editado en mapuzungun y en español, en una edición bilingüe. 
riores. Con eso suele callarse el niño; si no se tranquiliza, lo toma la madre junto con la cuna y así le da el pecho. De esta manera se crían las guaguas indígenas". (Pascual Coña, Lonco, 1995: 187-188) (...) "En la casa mapuche era persona principal el hombre de más edad; él mandaba a toda la gente que se encontraba en la ruca. La primera mujer del dueño de casa dirigía a las de su sexo". (Pascual Coña, Lonco, 1995: 189).

Los juegos eran eminentemente sociales, con un gran componente de desarrollo de habilidades físicas y sicomotrices. No hay ninguna descripción de juegos individuales $y$, en varios de los juegos colectivos, consideran competencias con premios aportados por los jugadores, que se ponen como objetos disponibles para el ganador.

Relata que de niño participaba en el trabajo productivo y de recolección de alimentos para la familia; en el proceso de siembra: "Cuando yo era chico todavía, ayudaba a mi padre. Él trabajaba en los cultivos del campo, sembraba un poco de trigo, otro poco de cebada, de arvejas, linaza, maíz, habas porotos, papas; sólo poco entraba de cada especie; los antiguos tenían poca práctica en esos trabajos". (Pascual Coña, Lonco, 1995: 30).

El trabajo infantil que relata se refiere a actividades domésticas ligadas directamente a la siembra, cuidado, recolección o extracción de alimentos vegetales, animales y frutos de mar o ríos. No se encuentra un trabajo productivo orientado al comercio o a los intercambios, sino fundamentalmente a la sobrevivencia del grupo familiar. El trabajo liviano es considerado una experiencia contextualizada de educación social orientada a preparar la condición de productor necesaria para la reproducción familiar y comunitaria.

Los niños y niñas se integraban a la sociedad también desde la espiritualidad. Narra extensamente el proceso que vivió su hermana, una niña de diez años, para cumplir su vocación de hacerse machi ${ }^{4}$, siendo dotada del conocimiento de los remedios y la oración. El padre se resistía a esta situación y no quería que fuese machi, pero ella se rebeló de la siguiente forma: "No digáis respecto de mí: 'Es el demonio que influye sobre ella'; no, el buen creador de la gente lo es, que va a hacerme machi para que sea persona afamada y cure los enfermos". (Pascual Coña, Lonco, 1995: 331).

Existe una visión de los niños como esencialmente buenos (inocencia natural) que se expresa con fuerza cuando dice: "que la admiren, como que es una niña tan pequeña, y digan 'buena machi será esta jovencita'. Si se tratara de una persona grande, podría engañar; pero siendo una niñita que se admiren" (...) "Tiene que hacerse machi nomás. Esa es una chiquilla todavía; si fuera mujer vieja, entonces se podría hablar de brujería, pero esa niñita no sabe nada de brujos. Seguro que el celestial creador de la gente la ha elegido para que sea una verdadera machi". (Pascual Coña, Lonco, 1995: 332-333).

Presenta una visión respecto de la carencia de toda maldad de los niños y reafirma que sus decisiones deben ser respetadas, siendo reforzadas por la comunidad,

4. Machi, varón o mujer que cumple funciones espirituales y de sanación en la comunidad; tienen un rol de alta importancia en la organización y tradición cultural y social mapuche. 
aunque, finalmente, se requiere también de la aprobación del padre, sin la cual las consecuencias pueden fatales para ésta. Es importante reconocer el alto valor que se le atribuye a la infancia en la dimensión de espiritualidad y sanación en la vida social comunitaria y como deben escucharse y respetarse las opiniones, intereses e incluso decisiones de los niños y niñas.

Se muestran algunas características interesantes de la relación familiar, y el papel de los niños en ella al enfrentar el proceso educativo formal (colonizador). Narra un ritual para integrar los niños al sistema escolar: se inicia cuando el educador (el sacerdote), solicita enviarlos a la escuela, a través de un mediador (capitán de amigos), a la máxima autoridad comunitaria (cacique y caciques subordinados), lo que pasa por la familia (abuela, madre) y termina en el niño, cuando se le pide consentimiento.

Se inicia en la escuela el proceso de educación formal y cristianización. En la escuela se les entrega comida y ropa, ya que entonces es con régimen de internado. Fritz (2006) resalta la importancia de los internados en este proceso de colonización a través de las Misiones, particularmente asociadas a una cierta noción moderna de preocupación por la pobreza. Se inicia en la misa y, según relata Pascual Coña, "en seguida me Ilevaron a la casa donde se lee y escribe: escuela se llama. El libro en que se aprenden las primeras letras (el silabario) sólo me lo entregaron dos días después". (Pascual Coña, Lonco, 1995:43). El aprendizaje se realizaba por la imitación de lo que hacían los niños que ya estaban en la escuela, por la repetición y enunciado de las letras.

En el territorio lafquenche del lago Budi y Puerto Saavedra, y respecto de la socialización y vida infantil tradicional, expresa una visión muy positiva de la infancia desde el nacimiento. En general, describe una infancia bastante feliz. La socialización inicial aparece predominantemente respetuosa de su che (ser-persona), como persona pequeña. Sin embargo, ella no está exenta de sufrir agresiones de adultos, como de ser robados en la comunidad o forzados a emigrar lejos de sus familias a internados o misiones.

La visión y narración de Pascual Coña es expresión de un nuevo y permanente mapuche: el de la interculturalidad. Hombres y mujeres que transitan entre dos culturas: entre la ciudad y lo rural, entre la comunidad y el distanciamiento, entre el español y la lengua vernácula mapuche, el mapuzungun (son bilingües), entre la cultura oral y la escrita, entre la vida en el lof (comunidad de una familia ampliada) y la vida en el internado, con su identidad transformada, sin dejar de ser lo que históricamente habían sido, con creencias propias, sincréticas o contradictorias.

\subsection{Ignacio Domeyko}

Ignacio Domeyko, sabio polaco, pilar del desarrollo científico de Chile, Rector de la Universidad de Chile, desde su visión etnocéntrica, científica, católica, europea y de funcionario del Estado chileno, relata sus observaciones de un viaje a la Araucanía en $1845^{5}$. (Domeyko, 1992a). Avecindado en Chile, es, como Pascual Coña, un

5. El libro expone dos textos de Domeyko que narran un viaje por la Araucanía en 1845. El primero (Domeyko, 1992a) organiza sistemáticamente el Diario de Viaje que corresponde al segundo libro, publicado recién en 1963 en Polonia (Domeyko, 1992b). 
hombre de la interculturalidad de la transición entre los siglos XIX y XX. En sus observaciones, realiza descripciones comentadas referidas a los procesos de vida y socialización de la infancia.

Observa en primer lugar que la familia es de gran importancia: "Sus casas son unos pequeños Estados que gozan de tanta independencia y respeto unas con relación a otras, como si fuesen capitales de distintas naciones. Todo en ellas está sujeto a las leyes y ceremonias antiguas: el umbral de la puerta es tan temible y sagrado como la frontera de un poderoso imperio". (Domeyko, 1992a: 87).

La familia es trasmisora y formadora de valores ancestrales y contemporáneos. Es la base de la comunidad y de la sociedad, en consecuencia donde se genera el "curriculum" formativo que recoge la historia local y colectiva y reproduce la lengua materna, según la lectura histórica del momento respecto de la lectura sobre la condición y tendencia de reproducción colectiva y de la definición de estrategias conscientes y espontáneas o locales de resistencia y construcción de condiciones de sobrevivencia material, social y cultural. La educación formal aunque no institucional como se ha referido antes y la socialización se ordenan en el territorio desde la familia a la sociedad.

Detalla extensamente los largos rituales de recepción y saludos a los viajeros, huéspedes y visitantes, donde se pregunta por los familiares: "Orden, severidad y disciplina parecen reinar en el interior de la familia: los hijos sumisos a sus padres, las mujeres ocupadas, unas en cuidar a sus chicos, otras en el servicio de la cocina, otras continuamente hilando la lana y tejiendo la ropa". (Domeyko, 1992a: 90).

Las mujeres (a las que considera más como "esclavas" de sus maridos que como libres) son las principales responsables de la crianza de los niños y del funcionamiento del hogar: "Son trabajadoras, pacientes, hacen todo el servicio de la casa, educan a los niños, hilan y tejen la ropa para esposo e hijos...". (Domeyko, 1992a: 90). El niño está íntimamente ligado a su madre, física y cotidianamente. Los infantes participan de la vida familiar, de los bailes, la música, la recepción a huéspedes y no tienen espacios diferenciados de los de los adultos, se socializan y educan en los espacios compartidos.

Las Misiones jugaron un papel formativo, en jóvenes varones y mujeres, que hoy son los abuelos de muchos de los adultos actuales y generaron procesos institucionales de ideologización, de instalación de una nueva racionalidad económica (campesinización y sub-proletarización), de integración forzada a la naciente nación chilena. Al mismo tiempo, instalaron un sistema escolar local en que se asociaba una escuela a un internado, lo que permitía que los niños, ya en pobreza, pudieran educarse, disciplinarse, ideologizarse las 24 horas del día. El internado, en el que trabajaban, asume en parte la tradición de participación económica de los niños en sus familias y comunidades y del trabajo como proceso formativo. Esta forma de sistema educativo local permanece hasta hoy, ya que la Araucanía cuenta con casi 18 mil niños y niñas internas (cifra de 2005).

En las descripciones de Domeyko, como también en las de Pascual Coña, la infancia aparece como una etapa de la vida en que a los niños no se les considera como minusválidos ("adulto menor o adulto pequeño"), sino como un momento del desarrollo con características propias, pero fuertemente ligadas a la vida familiar y comu- 
nitaria, en la que se manifiestan afectos y emociones. Su vida desde la primera infancia se desarrolla en el marco de una familia ampliada y de relaciones comunitarias intensas, con capacidad de tomar ciertas decisiones (que eran respetadas por los adultos), con juegos que les preparaban para las decisiones y competencias, participando de la vida económica a través del trabajo doméstico, integrados a la vida espiritual comunitaria e interesados en aprender cosas nuevas (como el catolicismo y la cultura chilena), lo que demostraba un interés por el conocimiento. Los adultos (padre y madre, abuelas, caciques, capitanes de amigos, machis, vecinos, parientes) son responsables de diversos momentos y situaciones de la socialización de los niños, algunos con funciones formativas específicas. Hay un currículo descentralizado en el que se forma la infancia, como proceso de selección y organización de contenidos culturales determinados desde la cultura y lengua, la historia de las comunidades y del pueblo, las condiciones de existencia, la visión de futuro y una pedagogía basada en la imitación, la práctica, la palabra y la conversación, el juego, el trabajo. Empieza la infancia y adolescencia a vivir la transculturación, producto de la colonización forzada, entre su mundo cultural y lingüístico trasmitido por la familia y comunidad y el que emerge desde las misiones, el comercio, los fuertes, las ciudades.

\section{AlgunOS ESTUDIOS SOBRE INFANCIA Y ADOLESCENCIA MAPUCHE}

Hay algunos estudios que dan cuenta de la situación de los niños y niñas mapuche actuales en la Araucanía que permiten actualizar la información disponible y contar con elementos de referencia y juicio para comprender las políticas sociales y de la educación intercultural bilingüe de niños, niñas y jóvenes mapuche en contextos indígenas e interculturales.

Fritz (2006) estudia las misiones en Boroa (próxima a la ciudad Temuco) con los Ilamados boroanos, de identidad mapuche-wenteche y las condiciones de vida, educación, traslado y percepciones familiares, sociales y religiosas respecto del proceso de aculturación religiosa. Muestra la ambigüedad de los procesos de socialización que viven los niños y niñas de fines del siglo XIX e inicios del XX. Explica que ellos se hallaban tensionados entre la pobreza resultante de las consecuencias de la guerra de expansión del Estado chileno y las opciones de sobrevivencia que les ofrecían las Misiones; entre la cultura que empezaba a redefinirse y la que se instalaba desde la fuerza; entre la socialización familiar-comunitaria y la escolar; entre la economía familiar sustentada en la sobrevivencia familiar y comunitaria y la economía mercantil; entre el trabajo libre y el servil o asalariado.

En el modelo educacional de la dominación los Internados ${ }^{6}$ actuales reproducen las Reducciones ${ }^{7}$ resultantes de la derrota militar ante el estado chileno. Los interna-

6. Internados: edificios anexos a establecimientos escolares donde estudiantes de origen rural o de pequeñas localidades, reciben servicios de alojamiento, alimentación, espacios para estudio y recreación durante los días hábiles de la semana o periodos más extensos.

7. Organización comunitaria rural impuesta por el estado luego de la conquista de La Araucanía a fines de la década de 1880. En ella se arrinconó a las familias en espacios cercados, con una relación personas/tierra y una condición productiva de agua/tierra desfavorable para una producción silvoagropecuaria de crecimiento y desarrollo. 
dos instalados en el territorio ancestral pese a la preocupación por la pobreza generada por la conquista reducían a los niños a un mínimo y extranjero territorio pedagógico, tal como sus familias eran reducidas a un mínimo territorio institucionalmente extranjero (aunque propio) de reproducción de la vida. El Internado educaba en la nueva cultura ideológica, la Reducción en la nueva cultura de producción de las condiciones de sobrevivencia, ambos en territorios reducidos, dependientes de otros, empobrecidos y empobrecedores de la cultura y estrategia de existencia propia y ancestral, donde la libertad de la palabra propia, la producción cultural de la identidad, la educación auténtica formal e informal, la creación tecnológica se cercenan radicalmente, se subordinan al poder dominante que se expandía por el territorio de los ancestros. Esta es una cuestión prácticamente no considerada en la EIB pese a su significado político e ideológico, pero también cultural, lingüístico y pedagógico. Así como las reducciones a la larga fueron espacios de resistencia, mantenimiento y renovación cultural y lingüística comunitaria hay que estudiar las posibilidades y amenazas que los Internados contienen respecto de los niños, niñas y jóvenes mapuche en las condiciones históricas y educacionales actuales y futuras.

Amigo, Bustos y Erazo (2000) analizan el crecimiento de los niños mapuche en relación a los no mapuche. Concluyen que, producto del mejoramiento de las condiciones de atención a las madres, los niños mapuche y no mapuche no nacen con diferencias sustantivas de peso o tamaño entre sí. Sin embargo, indican que ya al primer año se aprecia un desarrollo más lento en los primeros, lo que es atribuido a las peores condiciones de vida, es decir a la pobreza, particularmente en las zonas rurales. Complementariamente el Fondo de las Naciones Unidas para la Infancia (UNICEF) y el Ministerio de Planificación (Mideplan) elaboraron el Índice comunal de la infancia, que muestra que en aquellas comunas donde hay más presencia de población infantil o juvenil mapuche las condiciones de vida son más desfavorables en relación a ingresos, salud, educación y habitabilidad que en aquéllas en que la presencia indígena es menor o casi inexistente. (UNICEF/Mideplan, 2003). Los niños y niñas mapuche nacen en condiciones más desfavorables que aquéllos no indígenas, condición mitigada en el periodo previo y durante el nacimiento gracias a la intervención estatal en la salud materna, pero antes del primer año de vida éstas ya no son suficientes y nuevamente comienzan a pesar negativamente en las condiciones generales de vida.

Las condiciones de desarrollo local son desiguales según sea la pertenencia étnica. Esta desigualdad se expresa en las también inequitativas competencias personales desarrolladas para el ingreso al sistema escolar lo que coloca en discusión la necesidad de políticas de acción afirmativa, el papel del estado en la reducción de las inequidades, o las posibilidades reales que el mercado y la descentralización la superen.

Quidel (2005) desde una perspectiva cultural presenta una síntesis del aprendizaje en la cultura mapuche, en los procesos cognitivos y de socialización primaria. Distingue cuatro etapas del aprender comunes al ser humano: a) el acto de escuchar (Ajkütun): un ejercicio a través del que nos enteramos de las primeras nociones acerca de los fenómenos que debemos aprehender; es el primer ejercicio que desarrolla el ser humano como che. b) el acto de ver, de observar (Azkintun, pen, lelin): con un carácter de función visual, se refiere a la observación de los fenómenos que 
acontecen alrededor de las personas. c) iniciar la participación (Ina Konün zugu mew): es una etapa de experimentación de sensaciones, formas y estrategias que se inicia con lo lúdico, realizando actividades de imitación de los fenómenos escuchados e informados. Los términos referidos al kimün (conocimiento) se refieren a experiencias de orden más cognitivas en los ámbitos de rakizwam (actividad de pensar) y günezwam (experiencia de observar con profundidad los fenómenos). El rakizwam conduce al kimkonün que es el acto de lograr un aprendizaje, lograr un saber; el günezwam conduce al günekonün, que es un estado de tomar conciencia, de percatarse, un estado reflexivo acerca de lo que acontece alrededor; es volver a estar consigo mismo en forma consciente. d) y asumir la participación (Konün zugu mew): el participar en el acto mismo, hacerse parte de fenómeno, logra la práctica de lo escuchado, visto, recreado. Se siente en profundidad lo que se está realizando. Se ingresa a la experiencia y demuestra las habilidades para desarrollar la experiencia (Könuy zugu mew). Si lo demuestra, recibe el calificativo de kimüy (sabe) si no es así recibe el de kimlay (no sabe). (Quidel, 2005: 1-2).

Hay tres categorías del saber: zugun (el habla); az (carácter, personalidad, formas de ser); y pewman (el mundo de los sueños). En cuanto al conocimiento mapuche (kimvn), está presente en todo elemento que existe en el waj mapu; es algo vivo, que transforma, incita, despierta, seduce y conduce. Todo che tiene su propio kimvn, pues cada che es distinto, pues posee un az distinto y tiene un rol diferente en la vida. (Quidel, 2005: 2).

La calidad del che es una cualidad que se va desarrollando a lo largo de la vida y que supone varias dimensiones; a saber: $\tilde{N} i$ chegen: el ser persona; Kim chegen: ser sabio; Nor chegen: ser una persona recta; Übmeh chegen: ser una persona íntegra; Küme chegen: ser una persona buena. (Quidel, 2005: 3-4).

Quidel y Pichinao (2002) estudian a la infancia en tres territorios de identidades mapuche: lafkenche, wenteche y pewenche. Recorren algunas etapas y condiciones del desarrollo infantil como el embarazo, el tener un hijo, los niños, la territorialidad y las prácticas. Constatan un cambio drástico en los modos de educación propiamente mapuche que dan paso a un modelo de educación hispano-chileno, lo que resulta de la pérdida del control territorial a partir del 1880. Es muy drástico el paso del Xemümün, como una forma particular y un estilo de formación mapuche del che (a través del que se propician los sistemas valóricos propios del tuwün -territorio de origen-y del küpan -linaje- de las familias) a la forma escolarizada de educación en la cultura dominante. Señalan que hasta hace 30 años el modo de educación mapuche era funcional a la sociedad de entonces. El cambio generó consecuencias que provocan mayor vulnerabilidad ante el avasallamiento colonizante de la cultura dominante, lo que, a su vez, genera situaciones de desvinculación territorial que provocan quiebres internos y nuclearización de las familias, ente central en la formación de los niños, niñas y adolescentes a lo largo de su vida. Afirman que esos cambios están provocando una negación de la identidad mapuche y que hoy se constata una yuxtaposición de dimensiones de la vida social: atomizada concepción del ser humano y del mundo; religiosidad judeo-cristiana y espiritualidad propia; mapuzungun y lenguas extranjeras que no permiten desarrollar el orden social mapuche, sino fortalecen la sumisión al orden dominante. 
La escuela, según ellos, se ha enquistado en el territorio mapuche generando una serie de consecuencias, como: abandono de un modelo de formación propia; marginación de los niños y jóvenes de rituales socioculturales y religiosos; escolarización desde los jardines infantiles; Apropiación temprana de conocimientos no mapuche; privilegio del español por sobre el mapuzungun; incorporación de nuevos patrones de organización social no propios de la organización mapuche; exclusión del contexto cultural y geo-ecológico donde el niño nace, crece y se desarrolla. Señalan que su investigación demuestra la gran distancia valórica y de contenidos entre el modelo de educación mapuche y el hispano-chileno. Este último privilegiaría los contenidos que favorecen la competencia entre individuos y la formación de un intelectualismo que desconoce las experiencias vitales, afectividad y sentimientos que son medulares en la educación mapuche. Los adultos mayores mapuche hacen una fuerte crítica al modelo instalado, basado en su experiencia de vida; los jóvenes relativizan esa crítica por su menor experiencia en el modelo formativo mapuche y en el hecho de ya estar integrados a la cultura dominante.

Burrows y San Miguel (s.d.) describen las principales actividades que desarrollan, a lo largo de su vida, las niñas de la comunidad pewenche de Pitril, en Alto Bío Bío ${ }^{8}$. Presentan los modos de socialización familiar, expresándose en "chedungun" (manera en que la identidad mapuche pewenche denomina a la lengua vernácula que en otras identidades es denominada mapuzungun o de otras formas), en una concepción religiosa que combina catolicismo y creencias y prácticas tradicionales mapuche, donde existe una visión integrada de las diversas dimensiones de la vida social: religión, economía, estructura social, interpretaciones del medio natural. Por ejemplo, la niña, en sus roles de género, desde los 5 años es una colaboradora del hogar y cuando ingresa a la escuela debe pasar a cumplir dos roles distintos: en la escuela, para las profesoras, como alumnas; en el hogar, para la madre, como ayudante. Describen algunas características de la pedagogía familiar en la comunidad y destacan que el proceso de socialización se basa en la observación y en el hacer, más que en la transmisión de contenidos: las niñas y niños aprenden por sí mismos experimentando y demostrando destrezas. Los padres los entretienen con historias y ofrecen consejos, se mantienen las comunicaciones en chedungun, a pesar de que no se sostienen largas conversaciones en las reuniones familiares ni en los encuentros sociales, lo que haría que el desarrollo del lenguaje verbal pareciera ser más lento que en los niños no pewenche. Todas las niñas van a la escuela; los padres las llevan con poco convencimiento, de modo que si hay trabajos en el hogar o en el campo éstos son los privilegiados. Las madres esperan que la escuela refuerce los roles que se esperan de las mujeres y niñas en el hogar: buenas dueñas de casa a la usanza local, formación moral, legitimación de las normas comunitarias y familiares; los padres aspiran a que se reafirme su sistema de vida.

Díaz (1999) aborda el proceso de enseñanza-aprendizaje en el lof y en la escuela rural, buscando dilucidar diferencias entre ambos espacios formativos. Realiza un estudio de las modalidades de aprendizaje y los contextos en que se dan, que las

8. Estudio realizado antes de la radical transformación que sufrió el lugar por la construcción de dos grandes centrales hidroeléctricas en la zona (Pangue y Ralco). 
presenta in extenso, para concluir que la educación mapuche permite que todos los individuos puedan tener las mismas posibilidades de acceso a los conocimientos existentes, respondiendo así a sus necesidades e intereses propios; en cambio, la educación escolar responde a las necesidades de la sociedad capitalista reproduciendo sus objetivos y metodologías. El grupo familiar tradicional, con menor aculturación, responde a las necesidades de reproducción de su propia familia y comunidad, de su propia sociedad, para preparar a los individuos a que se integren a ella. Así, la herencia cultural se pone a disposición de cada uno y de todos. En estas familias los niños se educan a través de la observación del medio, la imitación, la participación, la responsabilidad, el trabajo y los juegos, actuando y realizando tareas concretas, manipulando, experimentando, sacando sus propias conclusiones. Es decir, aprenden en relación con las personas y en un clima de afectividad, lo que permite aprendizajes significativos y pertinentes culturalmente. Los niños se educan cerca de los adultos para ser guiados, orientados y motivados en una relación más horizontal que la que se produce en la escuela, pues en la educación participan miembros adultos de varias generaciones (padres, abuelos, tíos y otros parientes). La escuela desarrolla otros procesos educacionales y privilegia otros contenidos culturales; utiliza otros métodos pedagógicos y establece una relación social vertical. La autora no reniega de la escuela, plantea la necesidad de impulsar una transformación educativa desde la Educación Intercultural Bilingüe y de otros programas educacionales, como el de Educación Rural (MECE/Rural que ya no existe), que efectivamente asuman los principios de la educación mapuche.

La situación de los jóvenes es estudiada desde diversas perspectivas.

Díaz (1999) estudia también los procesos de formación de la identidad en jóvenes, mujeres, mapuche, en un internado urbano femenino de Temuco (zona de identidad wenteche, aunque las alumnas eran provenientes probablemente de otras identidades). Concluye que, si bien hay una autoadscripción como mapuche, hay también un proceso de integración de elementos de la cultura occidental, que las lleva a elegir aquellos elementos que consideran más útiles de una u otra cultura. Es en el internado donde se produce el paso de niña a joven, lo que les hace desenvolverse con más autonomía y desde ahí ir construyendo su identidad de género, construida desde la ciudad más que desde sus orígenes.

Valdés y Sanhueza (1996) estudian la migración campo-ciudad y sus consecuencias en jóvenes que llegan de la escuela básica rural al liceo urbano, en Nueva Imperial (de identidad wenteche). Concluyen que en este acto se conjuga una discriminación doble: por llegar del campo y por ser mapuche-rurales, en un fenómeno que se produce tanto entre docentes como alumnos y que afecta a la organización escolar (cursos separados o agrupaciones en las clases según los alumnos provengan o no de escuelas rurales), donde hay menores niveles de exigencia a los rurales que a los urbanos, y manifestaciones de racismo asociadas a expresiones prejuiciosas respecto del origen rural.

Romero (2000) estudia dos cohortes de jóvenes mapuche egresados del Liceo Humanista Científico de Ercilla (de identidad nagche): ninguno ha ingresado a la universidad; las mujeres están desempleadas o desempeñan labores hogareñas y los varones se encuentran trabajando en empleos menores, de baja calificación, tempo- 
reros y algunos desempleados. Sólo uno se encontraba estudiando una carrera de enseñanza técnica.

Gómez (2005) enfoca a los jóvenes desde otra perspectiva, conceptual y metodológica, en el mismo territorio de Ercilla. Estudia a un grupo de jóvenes del liceo de la ciudad que, en el Proyecto Kelluwün", se empodera por la participación de un proceso de Investigación Acción Participativa (IAP) que los lleva a determinar como problema crítico el que no tienen opciones de ingresar a la universidad, pues no están suficientemente preparados. Se organizan, diseñan un proyecto preuniversitario, consiguen el apoyo de un instituto, organizan los cursos y por primera vez consiguen que alumnos del liceo ingresen a la universidad.

Barría y Flores (2005) desarrollan otra IAP en la misma Comuna, junto a adolescentes mapuche internos (alumnos de identidad nagche entre $7^{\circ}$ año básico y $2^{\circ}$ de enseñanza media) del Liceo local. Realizan un diagnóstico realizado con los internos que mostraba timidez, silencio ante los adultos, baja autoestima, falta de identidad. Busca desarrollar competencias comunicativas al trabajar con la radio comunitaria Ercilla 100.3 FM. Al final del trabajo entre los jóvenes, las autoras, la radio y el internado se lograron avances en los adolescentes en relación a su capacidad comunicativa, identidad mapuche, autoestima y formación valórica. En medio del proceso surgieron problemas de violencia intrafamiliar, sexualidad, alcoholismo y violencia social, que se vivía en el mundo juvenil local con extensiones al liceo e internado.

Sin embargo, esta situación no es la común, ni tampoco la perspectiva de desarollo de los jóvenes una vez abandonada la enseñanza media.

Alvarado y Calabrano (2005) investigan la situación laboral de los egresados de la carrera de Técnico Agrícola de un Liceo de La Araucanía, en dos cohortes recientes, provenientes de territorios de diversas identidades. La situación no es diferente al estudio de Romero: la mayoría se encuentra o trabajando en actividades no vinculadas a su formación (en empleos precarios o temporales) o desempeñándose en predios familiares como trabajadores agrícolas. Una alumna estudiaba en la Universidad Católica de Temuco (Pedagogía Básica Intercultural). El estudio aborda la discriminación que sufren jóvenes por ser mapuche (la opinión de empleadores, según los entrevistados, es que los mapuche son conflictivos) y/o por ser mujer (según las alumnas, los empleadores no las contrataban pues consideraban que no tenían fuerza física para desempeñarse en labores agrícolas).

Los estudios de Romero y Alvarado y Calabrano (uno referido a la educación humanista-científico y otro a la técnico-profesional) muestran una realidad semejante: la dificultad que alumnos y alumnas mapuche tienen de insertarse en buenas condiciones en el mercado de empleo, donde las causas no estarían tanto en el tipo o calidad de la formación recibida sino en el contexto que no ofrece oportunidades, se toman decisiones de contratación sobre la base de prejuicios étnicos o de género. Si las condiciones de estudio expresan inequidades, en el tiempo generan efectos regresivos en la movilidad social considerando la variable étnica; estudios muestran que

9. El Proyecto Kelluwün impulsado, desde 1999, por la UFRO en el Municipio de Ercilla (La Araucanía), con comunidades locales e indígenas, impulsó la Educación Intercultural Bilingüe, Gestión Democrática de la Educación, EduComunicación y Participación Social. 
la movilidad (considerada como aumento sostenido de ingreso) es mayor para los no mapuche, media para los mestizos y menor para los mapuche expresando que la desigualdad étnica es institucionalizada y que la educación (con sus políticas de EIB) no consigue quebrar esa tendencia. (Cantero y Williamson, 2009).

Sepúlveda y Millaqueo (2004) desarrollan un trabajo de ayuda psicológica, social y cultural a niños y niñas de una comunidad afectada por el conflicto territorial que se desarrolla en la Araucanía. Sistematiza el trabajo Ilevado a cabo con 11 niños y niñas en la Comunidad José Guijón de Ercilla (de identidad mapuche-nagche). Muestran las graves consecuencias que el conflicto tiene en la violación de sus derechos y cómo no existe una preocupación estatal por protegerlos de los efectos sicológicos, escolares y emocionales que se producen en ellos, con manifestaciones psicosomáticas y culturales. Colombre, Gutiérrez, Saldaña y Torres (2004) estudian el mismo contexto, pero desde otra perspectiva: la de los jóvenes que se ven enfrentados a participar activamente del movimiento social mapuche de recuperación territorial en Ercilla y cómo conciben la trasgresión a las leyes del estado nacional mostrando las contradicciones que el sistema judicial tiene cuando se considera la variable cultural e intercultural en los asuntos de violación legal, juicio, sanción, reparación y reinserción.

\section{CONCLUSIONES}

El recorrido realizado a alguna literatura sobre infancia y socialización mapuche en la sociedad tradicional y actual de La Araucanía entrega algunas pistas de pautas de crianza y de educación que existían, permanecen o han desaparecido y que podrían ser consideradas en el diseño y ejecución participativa de las políticas públicas, programas de desarrollo y curriculum y pedagogía de la educación intercultural bilingüe (EIB) impulsadas en establecimientos educacionales con alta presencia escolar mapuche o en territorios comunitarios originarios, pero también, que puedan asumirse como aportes a la educación de escolares no mapuche y a los programas de interculturalidad mapuche-no mapuche.

La EIB no reúne hoy -no sabemos si podrá en alguna oportunidad futura dentro del mismo modelo económico neoliberal y democrático restringido de Chile- las condiciones para que la educación que reciben los(as) niños(as) y jóvenes mapuche puedan potenciar sus condiciones personales resultantes de su socialización inicial en la comunidad o familia, menos para que refuerce la cultura y contribuya a mantener la lengua. Los programas públicos han hecho esfuerzos por llevar adelante experiencias pedagógicas significativas y contextualizadas curricularmente, sin embargo su impacto no es suficiente para superar las desigualdades de acceso a los saberes de ambas culturas (la mapuche y la chilena o no mapuche) pues $-y$ no es tema de este artículo pero no por eso debe negarse- las condiciones generales societales de dominación y subordinación, de pobreza, exclusión y marginación económica, política, social, en que se encuentran los pueblos indígenas en relación a los no indígenas constituyen las principales razones de la invisibilidad, desconsideración o inadecuación de las acciones necesarias y pertinentes, participativas y efectivas, que aseguren calidad de la educación a los hijos e hijas indígenas, desde lo que deben aprender todos los estudiantes del país, lo que deben aprender como pro- 
pio los indígenas y todos como modo de convivir y cooperar en la sociedad global. Así no habrá ni aislamiento, exclusión o subordinación indígena que mata las culturas y lenguas poco a poco, pero tampoco habrá opresión, dominación, racismo o clasismo cultural desde la parte dominante de la sociedad. Ello supone comprender la función educadora como tarea de educadores profesionales y tradicionales que enseñan en sus respectivos territorios de aprendizaje, pero que, el aprendiz, vive como un proceso formativo en que hay continuidades, conexiones, complementos y articulaciones entre los saberes específicos de su dominio competente.

La educación no sólo debe basarse en el saber pedagógico acumulado en el sistema hegemónico sino en aquellos producidos y transmitidos en el sistema educacional de los pueblos originarios: no es el carácter mayoritario o minoritario de uso lo que otorga valor a los saberes sino el valor que éstos tienen en su propio mérito para mejorar la formación y la vida de los estudiantes. Nos muestra que el escenario de vida de los niños, niñas y jóvenes indígenas se ha modificado sustancialmente a lo largo de la historia de construcción de la coexistencia y convivencia intercultural, pero que su condición de subordinación, invisibilidad y exclusión permanece. La educación y la EIB para superar las inequidades actuales de acceso, permanencia, egreso en el sistema de educación escolar y superior y en consecuencia en la empleabilidad y movilidad social, debe diagnosticar en profundidad la variedad de situaciones específicas en que se manifiesta la discriminación, la desigualdad, la injusticia pedagógica y observar, valorar, reconocer y asumir contenidos culturales significativos y conceptos, principios, prácticas y recursos pedagógicos indígenas en la enseñanza: en un primer espacio pedagógico orientado a los(as) propios niños(as) mapuche y luego en el espacio de la interculturalidad ya que éste es un quehacer donde puede enriquecerse la formación desde y para ambas culturas.

La larga historia pedagógica del pueblo mapuche enseña que el niño, niña y joven, constituyen el centro del proceso de aprendizaje, que deben ser considerados como natural y esencialmente buenos; otorgándosele el derecho a ser personas pequeñas en formación con todas las posibilidades de desarrollar su vocación natural; la educación se inicia en la familia bajo su responsabilidad (con un papel rector de la madre) y se extiende a la comunidad y los intereses colectivos del pueblo o sociedad que es donde encuentra el propósito principal; se realiza en un territorio natural y cultural que se constituye en territorio de aprendizaje intercultural (Williamson, 2005); debe considerar una formación activa integral cognitiva, física, disciplinaria y espiritual; debe ser intercultural y plurilingüe, en una relación simétrica del aprendizaje de la lengua mapuche y del español; la pedagogía debe considerar: el modelamiento adulto, el trabajo productivo doméstico y liviano, el incentivo para el desarrollo permanente de la vocación natural y personal, la relocalización disciplinaria para el que enfrenta dificultades de aprendizaje en un área, el juego social, la conversación y el uso de la palabra, el ejercicio físico y la práctica auténtica; el curriculum debe seleccionar sus contenidos desde lo acumulado por la comunidad y lo presentado por la escuela para que sea intertranscultural (Padilha, 2004) y esté instalado en territorios históricos; los internados deben eliminarse y si no es posible deben reconstruirse para evitar que reproduzcan los principios dominadores de las antiguas reducciones indígenas. 
En tiempos en que los estándares homogenizan la educación hacia unidades de medida universales y en que poco a poco se toma conciencia de una profunda crisis de sentido y dirección de la escuela como sistema educacional que hace que la confianza en su capacidad de democratizar y contribuir a la movilidad social en la sociedad sea cada vez menor, quizás sea una oportunidad para aprender de aquellos pueblos y sujetos colectivos que han sostenido procesos educativos y culturales a lo largo de la historia, que ha sido de crisis, violencia, agresión, usurpación pero que ha permitido que los niños, niñas y jóvenes mantuvieran sentidos a sus existencias, contribuyeran a la existencia y reproducción colectiva, enriqueciera la cultura y lenguaje desde la interculturalidad y la ancestralidad, generara aprendizajes de competencias acordes a las necesidades de cada época, territorio, coyuntura y estrategia de desarrollo. ¿Será la educación, los decisores políticos, los educadores capaces de observar, aprender y apropiarse crítica pero positivamente de los éxitos pedagógicos de los pueblos originarios? La historia, la felicidad humana y de la naturaleza lo dirá y juzgará.

\section{REFERENCIAS BIBLIOGRÁFICAS}

ALVARADO ALVARADO, V. y CALABRANO JARA, F. (2005). Situación laboral actual de los(as) egresados(as) mapuche y no-mapuche de la modalidad técnicoprofesional agrícola del Complejo Educacional La Granja C-55, de la ciudad de Cajón, comuna de Vilcún. Cohortes generacionales 2001 y 2003. Tesis para optar al grado de Licenciatura en Educación. Temuco: Universidad de La Frontera.

AMIGO, H.; BUSTOS, P. y ERAZO, M. (2000). Niños mapuches. Crecimiento, nutrición y salud. Santiago: Universidad de Chile.

BARRÍA CORTEZ, P. J. y FLORES VALDÉS, L. E. (2005). Experiencia de Investigación Acción Participativa con jóvenes mapuche residentes en el Internado Alonso de Ercilla y Zúñiga de la Comuna de Ercilla. Tesis para optar al grado de Licenciatura en Educación. Temuco: Universidad de La Frontera/FEH/DE.

BURROWS, F. y SAN MIGUEL, J. (s.d.). La experiencia escolar de la niña pehuenche de Pitril, en el contexto de su socialización familiar y comunitaria. [Monografía inédita].

CANTERO, M. V. y WILLIAMSON, C. G. (2009). Movilidad social intergeneracional por origen étnico: evidencia empírica Región de la Araucanía, Chile. Universum, 24 (1), 22-40.

COLOMBRE S., C. N.; GUTIÉRREZ M., B. A.; SALDAÑA A., I. L. y TORRES, J. (2004). Adolescentes mapuche infractores de Ley Penal en el marco del "conflicto mapuche" en la provincia de Malleco. Tesis para optar al Título de Asistente Social. Temuco: Universidad Católica de Temuco.

DOMEYKO, I. (1992a). Araucanía y sus habitantes. Warszawa-Kraków: Sociedad Polaca de Estudios Latinoamericanos.

DOMEYKO, I. (1992b). Diario del viaje al país de los salvajes indios araucanos. En: Domeyko, Ignacio (1992), Araucanía y sus habitantes. Warszawa-Kraków: Sociedad Polaca de Estudios Latinoamericanos. 
DÍAZ COLIÑIR, M. (1999). El proceso de enseñanza aprendizaje en el lof y en la escuela rural. Temuco: Instituto de Estudios Indígenas/Universidad de La Frontera.

ERCILLA Y ZÚÑ̃IGA, A. de (1997). La Araucana (11ª ed.). Santiago: Editorial Universitaria.

FRITZ CARCAMÁN, J. J. (2006). La misión capuchina de Boroa, desde mediados del siglo XIX hasta principios del siglo XX. Tesis para optar al Título de Profesora de Historia, Geografía y Educación Cívica y al Grado de Licenciada en Educación. Temuco: Universidad de La Frontera.

GÓMEZ, R. P. (2005). Experiencia sobre empoderamiento educacional juvenil. El caso de Ercilla. Tesis en preparación (Magister en Educación). Santiago: Universidad Metropolitana de Ciencias de la Educación.

PADILHA, P. R. (2004). Curriculo Intertranscultural. Novos itinerarios para a educacao. Sao Paulo: Cortez Editora, Instituto Paulo Freire.

PASCUAL COÑA, L. (1995). Lonco Pascual Coña ñi tuculpazugun. Testimonio de un cacique mapuche (5aㅡ. ed.). Santiago: Pehuén.

QUIDEL, J. (2005). De cómo aprenden los adultos mapuche (Chumgechi ñi kimken wünenke xem pu mapuche). Documento de Curso de Educación de Adultos. Temuco: Universidad de La Frontera.

QUIDEL, J. y PICHINAO, J. (2002). Haciendo crecer personas pequeñas en el pueblo mapuche. Temuco: Secretaría Ministerial de Educación Región de La Araucanía.

ROMERO, J. M. (2000). Situación actual de hombres y mujeres, mapuche y no mapuche, egresados del Liceo H-C Alonso de Ercilla de la comuna de Ercilla (Cohortes generacionales 1997 y 1999). Tesis para optar al grado de Licenciado en Educación. Temuco: Universidad de La Frontera/FEH/DE.

SEPÚLVEDA, R. y MILLAQUEO, A. (2004). Informe de diagnóstico e intervención en la comunidad Cacique José Guiñón. Angol: Servicio de Psiquiatría del Hospital de Angol.

VALDÉS, J. y SANHUEZA, E. (1996). La inserción de estudiantes rurales mapuche en escuelas urbanas: discriminación, autoestima e identidad. Tesis para optar al grado de Licenciado en Educación. Temuco: Universidad de La Frontera/FEH/DE.

WILLIAMSON, G. (2005). Territorios de aprendizajes interculturales: transitando a una nueva praxis pedagógica. Pensamiento Educativo, 37, 163-181. 